\title{
MODEL OF A DISK-OUTFLOW COUPLED SYSTEM: DISK-OUTFLOW SYMBIOSIS
}

\author{
BANIBRATA MUKHOPADHYAY \\ Department of Physics, Indian Institute of Science, Bangalore 560012 \\ E-mail:bm@physics.iisc.ernet.in
}

\begin{abstract}
Several observational evidences and deeper theoretical insights reveal that accretion and outflow/jet are strongly correlated. We model an advective disk-outflow coupled dynamics. We investigate the properties of the disk-outflow surface and how is it dependent on the spin of the black hole. The energetics of such a symbiotic system strongly depend on the mass, accretion rate and spin of the black holes. The model clearly shows that the outflow power extracted from the disk increases strongly with the spin of the black hole.
\end{abstract}

Keywords: accretion: accretion disk — black hole physics - galaxies: active - galaxies: jets — gravitation — hydrodynamics — relativity — X-rays: binaries

\section{Introduction}

There are many observational evidences for strong outflows and jets in black hole accreting systems, both in microquasars (e.g. SS433, GRS 1915+105) ${ }^{11}$ and quasars or active galactic nuclei (AGNs) (e.g. Sgr $\left.A^{*}\right){ }^{2]}$ Extragalactic radio sources show evidences for strong jets $\frac{[3}{4}$ As the jets are coming from the disk, base of the jets can not be treated independent of disk, which might be influenced by the spin of black holes. Outflows/jets take matter out of the disk, which helps in removing angular momentum of the disk and infalling the matter. Hence, inflow/disk and outflow/jet governing from a same system can not be treated independently. Indeed, observed data argue for the correlation of radio to X-ray emissions. ${ }^{4}$

Most of the jet emissions are associated with the low/hard state of accretion disk (e.g. GRS 1915+105). On the other hand, a low/hard state corresponds to a non-Keplerian flow ${ }^{[5}$ Hence, here we report the coupled disk-outflow dynamics in the framework of a sub-Keplerian, advective, geometrically thick accretion disk. For a detailed description, see Ref. 6.

\section{Basic equations}

We consider the correlated dynamics, governed by conservation laws, based on bi-directional hypothesis: outflow/jet is necessary to satisfy disk boundary condition(s). Hence, we plan to solve the set of coupled partial differential hydrodynamic equations in the advective regime, when flow variables depend on radial $(r)$ and vertical $(z)$ coordinates. However, we neglect the effects of magnetic field, as we do not aspire to describe the jet mechanism. Moreover, there are models (CENBOL, ${ }^{7}$ $\mathrm{ADAF}^{8}$ ), arguing for outflows without magnetic effects. Indeed, supercritical accretions predict outflows/jets by strong radiation pressure. ${ }^{9}$ In addition, we neglect viscosity as we concentrate on a predefined inner region of the system where outflowing matter is able to remove angular momentum and then transport matter. 
Hence, the hydrodynamic balance equations along with the equation of continuity in the pseudo-Newtonian framework are give by

$$
\begin{aligned}
& \frac{1}{r} \frac{\partial}{\partial r}\left(r \rho v_{r}\right)+\frac{\rho v_{z}}{z}=0, v_{r} \frac{\partial v_{r}}{\partial r}+v_{z} \frac{v_{r}-v_{r 0}}{z}-\frac{\lambda^{2}}{r^{3}}+F_{G r}+\frac{1}{\rho} \frac{\partial P}{\partial r}=0, \\
& v_{r} \frac{\partial v_{z}}{\partial r}+\frac{v_{z}^{2}}{z}+F_{G z}+\frac{1}{\rho} \frac{P-P_{0}}{z}=0, \frac{d \lambda}{d t}=0,
\end{aligned}
$$

where we choose $\partial / \partial z \equiv 1 / z$ as the radial variations of the underlying variables are much stronger than their vertical variations so that the flow structure remains disk. Here $v_{r}, v_{z}, \lambda, \rho, P$ define respectively radial, vertical velocities, specific angular momentum, density, pressure of the flow and those with subscript ' 0 ' are the respective variables at $z=0$. The above equations are to be solved with the condition $\left.P\right|_{h}=0$, when $h$ is the disk scale height, applying in the vertical momentum balance equation at $z=h$ and by the prescription of two-dimensional pseudo-Newtonian potential given by Ref. [10. We also assume $P=K \rho^{\gamma}$, where $K$ is a constant and $\gamma$ the adiabatic index, and the sound speed $c_{s}=\sqrt{\gamma P / \rho}$. Presuming that the outflow velocity is not likely to exceed the sound speed at the disk-outflow surface, we further propose $v_{z}=l(z / r)^{\mu} c_{s}$, when $l$ and $\mu$ are the constant parameters.

\section{Solutions}

Solving the set of eqns. (11), we find the disk-outflow coupled region ceased to exist at a $z=h$ where $v_{r}=0$, as outflow takes away the matter. Figure 19 shows the upper surface of the coupled region $\left(h_{\text {surf }}\right)$. The arrows in the diagram reveal the direction of flow. A more realistic disk-outflow surface could be visualized with a thick-solid line around the peak shown in Fig 1]. Figure 10 shows the geometry of the entire disk-outflow coupled region and that how the region changes with the change of spin of the black hole $(a)$. Larger the spin, thinner the region is, and advancing the peak of the region towards the black hole (i.e. the system becomes more prone to produce outflows in the vicinity of black hole). This is because, a faster spinning black hole is more prone to eject matter out leaving a smaller remnant. Additionally, stronger centrifugal effect of a faster spinning black hole ejects matter from a closer vicinity of the black hole compared to a slower black hole. Figure 1, shows the variation of the radius ceasing the disk-outflow coupled region in the vicinity of the black hole $\left(R_{j t}\right)$ with $a$, reflecting the same feature mentioned above. Finally, Fig. 1 1 d shows clearly the increase of outflow (and hence jet) power with increasing $a$. Note that the mechanical outflow power is defined as $P_{j}(r)=\left.\int 4 \pi r\left[\left(\frac{v^{2}}{2}+\frac{\gamma}{\gamma-1} \frac{P}{\rho}+\phi_{G}\right) \rho v_{z}\right]\right|_{h_{\text {surf }}} d r$, where $v$ is the magnitude of total velocity and $\phi_{G}$ the gravitational potential.

\section{Conclusions}

It is found that the power of astrophysical jets increases with the increasing spin of central object. In case of blazars, emissions are believed to be originated from their 

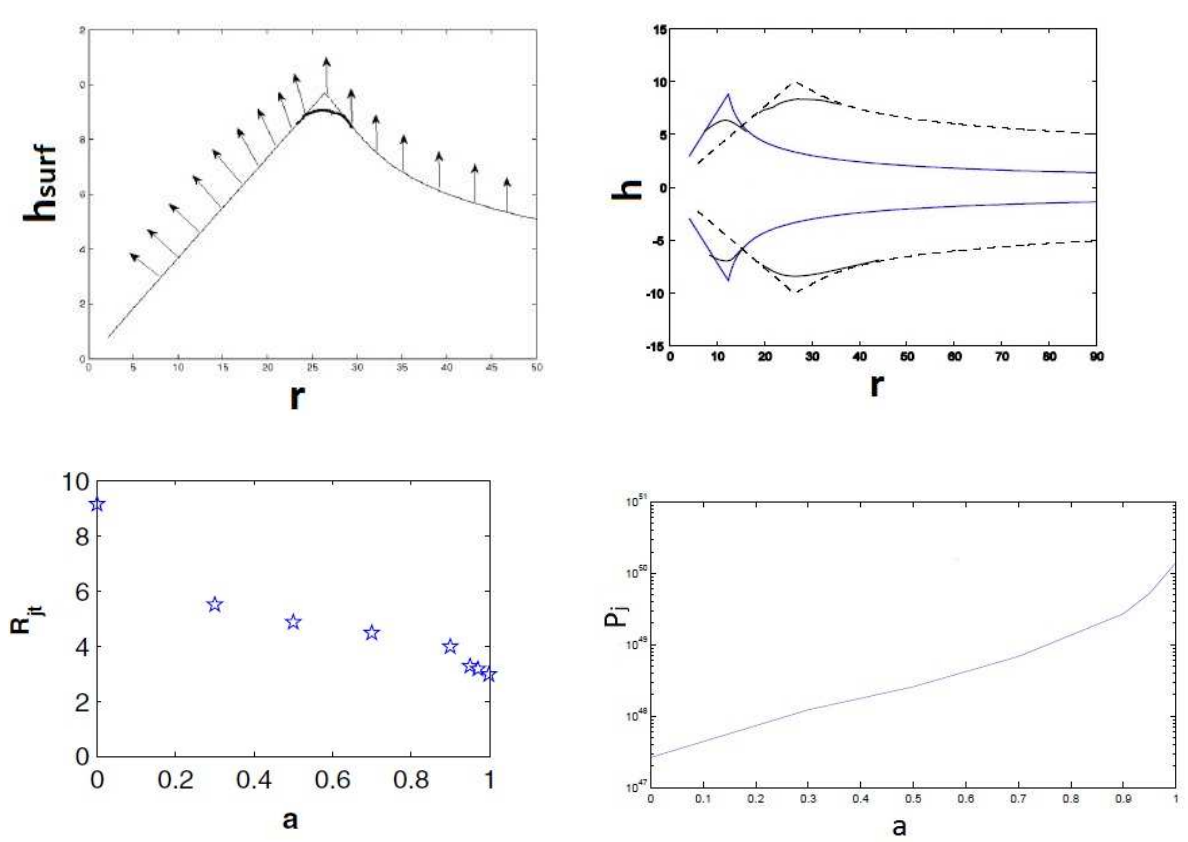

Fig. 1. Upper-left (a): Nature of the surface of disk-outflow coupled region. Upper-right (b): Geometry of disk-outflow coupled region, solid and dashed lines are respectively for $a=0.998$ and 0 . Lower-left (c): Variation of inner radius ceasing disk-outflow coupled region as a function of black hole's spin. Lower-right (d): Outflow power in C.G.S. unit as a function of black hole's spin, with one Eddington accretion rate around a $10^{7}$ solar mass black hole.

jets, whose high energy properties can be understood from this theory; see Ref. 11. If the extreme gravity is responsible for powering strong outflows and jets, then the spin of the black hole, perhaps, is the fundamental parameter to account for the observed astrophysical processes.

This work was partially supported by the grant ISRO/RES/2/367/10-11.

\section{References}

1. I. F. Mirabel \& L. F. Rodriguez, Nature 371, 46 (1994).

2. M. C. Begelman, R. D. Blandford \& M. J. Rees, RvMP 56, 255 (1984).

3. D. L. Meier, K. Shinji \& Y. Uchida, Science 291, 84 (2001).

4. R. P. Fender, T. M. Belloni \& E. Gallo, MNRAS 355, 110 (2004).

5. S. L. Shapiro, A. P. Lightman \& D. M. Eardley, ApJ 204, 187 (1976).

6. D. Bhattacharya, S. Ghosh \& B. Mukhopadhyay, ApJ 713, 105 (2010).

7. S. K. Chakrabarti, $A \& A$ 351, 185 (1999).

8. R. Narayan \& I. Yi, ApJ 428, 13 (1994).

9. M. A. Abramowicz \& T. Piran, ApJ 241, 7 (1980).

10. S. Ghosh \& B. Mukhopadhyay, ApJ 667, 367 (2007).

11. B. Mukhopadhyay, this volume. 